\title{
The Effectiveness of Real-Time Ultrasound-Guided Central Venous Catheterization: A Comparison with the Landmark Technique in Jordanian Patients
}

\author{
${ }^{1}$ Amjad Bani Hani, ${ }^{2}$ Amjad Shatarat, ${ }^{1}$ Rami Ayoub, ${ }^{3}$ Baeth Moh'd Alrawashdeh, \\ ${ }^{1}$ Amir Malkawi, ${ }^{1}$ Zahraa Al-Tamimi, ${ }^{1}$ Moaath Alsmady, ${ }^{4}$ Isam Bsisu and ${ }^{1}$ Mahmoud Abu Abeeleh \\ ${ }^{1}$ Department of General Surgery, School of Medicine, University of Jordan, Amman, Jordan \\ ${ }^{2}$ Department of Anatomy and Histology, School of Medicine, University of Jordan, Amman, Jordan \\ ${ }^{3}$ Department of Special Surgery, School of Medicine, University of Jordan, Amman, Jordan \\ ${ }^{4}$ Department of Anaesthesia and Intensive Care, School of Medicine, \\ University of Jordan, Amman, Jordan
}

\begin{abstract}
Real-time ultrasound-guided Central Venous Catheterization (CVC) has been demonstrated to reduce insertion and procedure time as well as having less complications when compared to the landmark-guided technique. This technique was newly introduced to Jordan University Hospital (JUH) and the aim of this study is to report our experience in both techniques. Medical records of 203 patients who needed central venous catheterization in the JUH between May, 2016 and May, 2017 were reviewed. The ultrasound-guided group included 102 patients while the landmark-guided group included 101 patients. The duration of catheter insertion, procedure duration and complications were considered. The average number of catheterization attempts in the ultrasound-guided group was significantly less when compared with the landmark-guided group $(p<0.001)$. Furthermore, the duration of insertion and the duration of the procedure were both significantly shorter in the ultrasound-guided group compared to the landmark group ( $\mathrm{p}<0.001$ for both). Carotid artery punctures and hematoma formation were also significantly less in the ultrasound-guided group compared with the landmark group $(\mathrm{p}=0.019)$. The ultrasound-guided method for central venous catheterization could increase the efficiency and reduce medical complications which justifies the costs of implementing this technique.
\end{abstract}

Key words: Landmark, ultrasound, central venous catheter, internal jugular vein, ultrasonography, reduce

\section{INTRODUCTION}

Central Venous Catheterization (CVC) is a commonly used procedure in the operating theatre in the intensive care unit in elective or urgent procedures and at medical or surgical wards (Emerman et al., 1990). The commonly used site for CVC placement is the internal jugular, subclavian and Femoral Veins (FV) (Palepu et al., 2009). This procedure is frequently used for hemodynamic monitoring, long-term administration of fluids, antibiotics, chemotherapy and hemodialysis (Koski et al., 1992; Turker et al., 2009). The CVC in the preferred vein is performed either by using the landmark method which is a blind procedure using anatomical landmarks on the skin's surface and thus passing the needle along the anticipated line of the vein or by using UltraSound-guided (US) method.

The success of landmark guided CVC depends on patient anatomy, comorbidities and physician experience (McGee and Gould, 2003; Scott, 1988). Moreover, 9\% of patients have anatomical variations that result in complications when using the landmark assisted central venous line insertion method (Denys et al., 1993), so that, many immediate and delayed complications have been reported (Scott, 1988). Immediate complications include artery puncture, hematomas, pneumothorax and hemothorax while delayed complications include infection and thrombosis (Digby, 1994; Sznajder et al., 1986).

Due to these complications, real-time ultrasound guided CVC was introduced in 1986 (Yonei et al., 1986). Following that different modalities of ultrasound-guided CVC have been used to decrease complications associated with central line insertion, improving the success rates, reducing the time required to perform the procedure and lowering the costs (Denys et al., 1993; Randolph et al., 1996).

The ultrasound guided technique was newly introduced to Jordan University Hospital (JUH) and up to our knowledge, Landmark guided techniques have not been compared to ultrasound guided methods in Jordan. Our aim in this study is to determine whether ultrasound guided central venous line, insertion is superior to landmark-guided techniques in the setting of the Jordan 
University Hospital and to describe the experience regarding placement of land mark of CVCs or ultrasound-guided CVCs by our physicians. The variables of interest in this study are the number of successful catheterizations, the number of attempts until successful catheterization, the duration of insertion (time from fist puncture till the guide wire was inserted in the vein) and the rate of complications.

\section{MATERIALS AND METHODS}

Study population: This retrospective cohort study was conducted at JUH which is located in Amman, the capital of the Hashemite Kingdom of Jordan. The study included 203 patients who needed a central venous access in the Intensive Care Unit (ICU) and in the Operating Theatres (OR) from May, 2016 till May, 2017. Due to the availability of the ultrasound machine in the OR, patients undergoing $\mathrm{CVC}$ at the $\mathrm{OR}$ were enrolled in the UltraSound group (US) and ICU patients undergoing CVC were enrolled in the LandMark group (LM). The age, weight, height, Body Mass Index (BMI), abdominal girth, International Normalized Ratio(INR), hemoglobin levels, hematocrit and platelet count was obtained for the patient from the medical record, procedural notes and nurse notes. The mechanical complications such as carotid artery puncture and hematoma formation were recorded for both group.

Landmark technique: The patients were placed in a supine position. The skin between the two heads of the right sternocleidomastoid muscle was cleaned with acetone and povidone-iodine. Xylocaine (1\%) was used as a local anesthetic and was administered through a 22-gauge needle. This 22-gauge needle was inserted at a $45^{\circ}$ angle and used to locate the Internal Jugular Vein (IJV). Backflow of venous blood into the syringe confirmed entry of the finder needle. The syringe was removed and the guidewire was inserted. Once the guidewire was correctly positioned, the needle was removed. The vascular access device/central venous catheter was placed over the guidewire and into the IJV. real-time ultrasound-guided method.

Patients were prepared in the same way as the LM group as described above. The outlay ultrasound technique was used in all cases. The probe was covered with ultrasonic gel and draped with a sterile plastic sheath. Standard ultrasound Two-Dimensional (2D) imaging was used. If a thrombus was detected or in the case of failure to gain access, the contralateral side was catheterized. If the trial on the contralateral side was not successful as well, the subclavian vein was catheterized, followed by the femoral vein.

A 19-gauge, $10 \mathrm{~cm}$ needle was advanced under the skin under the guidance of the ultrasound probe. The following steps are the same as those used in the landmark group and no special catheterization equipment was used for the ultrasound group. All ultrasound and landmark guided catheterizations were performed by experienced senior residents or specialists.

Ethical considerations: The study was approved by the Ethics Committee and Institutional Review Board (IRB) at Jordan University Hospital Ethics Committee. The study was conducted in compliance with the ethical standards of Jordan University Hospital Ethics Committee and the Helsinki Declaration and all collected data were solely used for statistical analysis.

Data collection: Data was collected and recorded in a timely fashion. Patient's age, gender, height, weight, BMI, abdominal girth, INR, hemoglobin and platelet count was recorded. In addition, the vein catheterized and the site of catheterization (right or left) were recorded. We recorded whether catheterization was successful, the number of attempts at catheterization until success, duration of insertion, duration of procedure, carotid artery puncture, hematoma formation, previous medical conditions and whether a specialist was needed to perform the catheterization. The duration of insertion was defined as the time from puncture of the skin until the wire was in its designated position. The duration of the procedure was defined as the time from puncturing the skin until the device/catheter was in its final position. These times were measured in seconds by a team member.

Statistical analysis: SPSS Software vJanuary 8, 2020ersion 20 (SPSS Inc., Chicago, IL, USA) was used for data entry and analysis. Continuous variables were presented as a mean $\pm \mathrm{SD}$ while categorical variables were presented as a number of patients per category. To identify the differences between the two groups, we used independent sample $t$ tests for continuous variables and $\chi^{2}$ test for categorical variables. p-values $<0.05$ were considered for statistically significant correlations.

\section{RESULTS AND DISCUSSION}

This study involved 203 patients (who needed a central venous Access) and it was divided into two groups. Of these patients, $102(50.2 \%)$ underwent an ultrasound assisted CVC while 101 (49.8\%) had an anatomical landmark assisted insertion. Table 1 shows the baseline characteristics of the study population. The mean age was $57.1 \pm 20.7$ years for the US group and $54.9 \pm 18.3$ years for the LM group with no significant difference. In addition, there were no significant differences between the two groups of patients in gender, BMI, waist circumference having diabetes or AKI (Table 1). 
Res. J. Med. Sci., 13 (6): 109-114, 2019

Table 1: Demographics and baseline characteristics of the study population

\begin{tabular}{|c|c|c|c|}
\hline Variables & Landmark group overall $(\mathrm{N}=101)$ & Ultrasound group overall $(\mathrm{N}=102)$ & $\mathrm{p}$-values \\
\hline \multicolumn{4}{|l|}{ Gender } \\
\hline Female & $41(40.6)$ & $35(34.3)$ & 0.355 \\
\hline Male & $60(59.4)$ & $67(65.7)$ & \\
\hline \multicolumn{4}{|l|}{ Age (years) ${ }^{a}$} \\
\hline \multirow[t]{2}{*}{ Range } & $57.1 \pm 20.7$ & $54.9 \pm 18.3$ & \\
\hline & $(3.8-98.1)$ & $(18.1-88.1)$ & 0.410 \\
\hline $\mathrm{BMI}\left(\mathrm{kgm}^{-2}\right)^{\mathrm{a}}$ & $27.3 \pm 4.8$ & $26.3 \pm 3.3$ & 0.105 \\
\hline Waist circumference $(\mathrm{cm})^{\mathrm{a}}$ & $81.4 \pm 14.2$ & $80.8 \pm 10.3$ & 0.721 \\
\hline \multicolumn{4}{|l|}{ Approach } \\
\hline Internal jugular & $79(78.2)$ & $100(98.0)$ & $<0.001$ \\
\hline Subclavian & $10(9.9)$ & $0(0.0)$ & \\
\hline Femoral & $12(11.9)$ & $2(2.0)$ & \\
\hline Specialist (yes) & $9(8.9)$ & $24(23.5)$ & 0.005 \\
\hline Resident (yes) & $92(91.1)$ & $78(76.5)$ & 0.005 \\
\hline Diabetes (yes) & 44 (43.6) & $57(55.9)$ & 0.079 \\
\hline Hypertension (yes) & $60(59.4)$ & $66(64.7)$ & 0.436 \\
\hline ESRD (yes) & $4(4.0)$ & $62(60.8)$ & $<0.001$ \\
\hline AKI (yes) & $9(8.9)$ & $14(13.7)$ & 0.279 \\
\hline Hemoglobin $\left(\mathrm{mgdL}^{-1}\right)^{\mathrm{a}}$ & $11.2 \pm 1.9$ & $10.3 \pm 1.9$ & 0.002 \\
\hline $\mathrm{INR}^{\mathrm{a}}$ & $1.15 \pm 0.1$ & $1.11 \pm 0.1$ & 0.038 \\
\hline Platelet $^{\mathrm{a}}$ & $257.0 \pm 122.0$ & $228.9 \pm 101.0$ & 0.076 \\
\hline
\end{tabular}

${ }^{a}$ Values are presented as mean \pm standard deviation and n (\%). BMI: Body Mass Index; ESRD: End-Stage Renal Disease; AKI: Acute Kidney Injury; INR: International Normalized Ratio

Table 2: Outcome measures in the ultrasound group versus the landmark group of patients

\begin{tabular}{lccr}
\hline Outcomes & Landmark group overall $(\mathrm{N}=101)$ & Ultrasound group overall $(\mathrm{N}=102)$ & $\mathrm{p}$-values \\
\hline Success rate & $98(97.0)$ & $102(100)$ & 0.314 \\
Average number of attempts & $1.98 \pm 1.1$ & $1.29 \pm 0.5$ & $<0.001$ \\
Mean \pm SD & & & \\
Average duration of insertion (minutes), mean $\pm \mathrm{SD}$ & $6.1 \pm 4.3$ & $2.9 \pm 1.4$ & $<0.001$ \\
Hematoma (Yes) & $4(4.0)$ & $0.0(0.00)$ & 0.042 \\
Carotid injury (Yes) & $12(11.9)$ & $2(2.0)$ & 0.005 \\
\hline
\end{tabular}

${ }^{\mathrm{a}}$ Values are presented as mean \pm standard deviation and $\mathrm{n}(\%)$

Table 3: Descriptive statistics for performing CVC between experienced and inexperienced physicians

\begin{tabular}{lccc}
\hline Outcomes & Average number of attempts & Average duration of insertion & Average duration of procedure \\
\hline User experience in landmark method & & & \\
Resident & $1.92 \pm 1.14$ & $6.02 \pm 4.3$ & $18.30 \pm 6.1$ \\
Specialist & $2.55 \pm 1.23$ & $6.88 \pm 4.1$ & $18.30 \pm 6.1$ \\
p-value & 0.119 & 0.566 & 0.989 \\
User experience in ultrasound method & & & \\
Resident & $1.29 \pm 0.5$ & $3.04 \pm 1.5$ & $15.33 \pm 4.3$ \\
Specialist & $1.29 \pm 0.6$ & $2.58 \pm 1.3$ & $15.08 \pm 6.4$ \\
p-value & 0.979 & 0.184 & 0.827 \\
\hline
\end{tabular}

${ }^{\mathrm{a}}$ Values are presented as mean \pm standard deviation and $\mathrm{n}(\%)$

The most common route of insertion in both groups was the IJV, followed by femoral vein and then the subclavian vein $(\mathrm{p}<0.001)$. Two hundred out of the 203 procedures were performed successfully $(98.5 \%)$. The three failed catheterizations were all among the LM group. The difference between the success rates of the two groups is not statistically significant as shown in Table 2.

Our results show a statistically significant difference between the mean number of attempts at inserting the central venous line between the LM $(2 \pm 1.2)$ and US $(1.3 \pm 0.5)$ groups $(p<0.001)$. Furthermore, the duration of insertion in the US group was $2.9 \pm 1.5 \mathrm{~min}$ and the duration of the procedure was $15.3 \pm 4.9 \mathrm{~min}$. In comparison, the LM group had an average duration of insertion of $6.1 \pm 4.3 \mathrm{~min}$ and the duration of the procedure was $18.3 \pm 6.2$. The difference between the durations of insertion was significantly different between the two groups $(p<0.001)$. The difference between the duration of the procedures was also statistically significant $(\mathrm{p}<0.001)$.

Upon investigating the required experience for CVC, $23.5 \%$ of procedures in the US group were performed by a specialist, compared to $8.9 \%$ among LM group $(p=0.005)$. Similarly, more residents performed landmark technique (91.1\%), compared to $76.5 \%$ for US guided technique $(p<0.005)$. However, no significant differences were found between resident physicians and specialists in the number of attempts, duration of insertion and total duration of procedure (Table 3 ). 
The overall complication rate was higher in the LM group compared to the US group. The most frequent complications in the LM group compared to the US group were the carotid puncture and hematoma $(p<0.005)$. Remarkably, $98 \%$ of patients in the US group had no complications, compared to $88 \%$ in LM group (Table 2).

Central venous catheterization is a widely used procedure in hospitals and it can be performed through peripheral and central veins. The CVC is used to for administration of fluids, long-term medication, haemodialysis and to administer chemotherapy (Karakitsos et al., 2006).

In the LM group in orderto determine the puncture site on the skinvisible and palpable external landmarks with known relation to the targeted vessel were used (Bannon et al., 2011). The percent of failure with the landmark method can reach 35\% (Sznajder et al., 1986). Unfortunately, this method is associated with several complications that may lead to an increase in morbidity, expenses, longer hospital stay and mortality (McGee and Gould, 2003). Complications are usually divided into mechanical $(5 \%$ up to $19 \%)$ of patients, infectious complications (5\% up to $26 \%$ ) and thrombotic complications ( $2 \%$ up to $26 \%$ ) of patients (Ge et al., 2012; Richards et al.,1999).

Using ultrasound-guided catheterization improves success rates and decreases the rates of complication rates compared to the landmark-guided approach (Hind et al., 2003; Wu et al., 2013). The present study was the first in Jordanto assess the efficacy of using the ultrasound guided technique in the Jordanian population.

This current study has shown that there was no statistical significant difference between the success rates of the US group and the LM group $(p=0.314)$. These results are consistent with the findings of Turker et al study (Turker et al., 2009). However, other studies have demonstrated an increased rate of successful catheterizations in the US group when compared with the LM group (Karakitsos et al., 2006; Sazdov et al., 2017; Serafimidis et al., 2009). This contradictory between our results and otherscould be attributed to the fact that at JUH usually trained senior residents insert central venous catheters, reducing failure rates. Using landmark method, we found that the overall success was $97 \%$ that is consistent with other reports (ranging from $85-100 \%$ ) (Fragou et al., 2011; Karakitsos et al., 2006; Prabhu et al., 2010).

One of our objectives in this present study was to determine the number of attempts needed to place CVC between the US-guided and LM methods. Our data have shown that US-guided method required less attempts for successful catheterization than the LM technique. These results are in line with facts that the use of US guidance for $\mathrm{CVC}$ allows for detailed anatomical visualisation of the veins and surrounding structures, thus reducing the number of attempts needed (Serafimidis et al., 2009). Several studies have demonstrated that US guidance required fewer attempts for successful catheterization than the LM technique (Hosokawa et al., 2007; Miller et al., 2002; Serafimidis et al., 2009).

Duration of CVC insertion was also measured in our study where it has been demonstrated in US-guided group the duration was less than in the LM group. This significant reduction in time needed to insert the catheter is similar to what has been reported in previous studies (Denys et al., 1993; Hosokawa et al., 2007; Miller et al., 2002). In addition, our study has demonstrated that the US-guided method has markedlyreduced the time needed to perform the whole procedure $(\mathrm{p}<0.001)$. These results are in agreement with what Troianos et al. (1991) have reported that all central venous catheters were inserted within 3 min when using the ultrasound, a figure close to the average of 2.9 min needed in our study (Troianos et al., 1991).

Another important objective of our study was to determine the complication rate between the LM and US-guided methods. Data obtained in our study have indicated that US-guided method resulted in significantly less complications compared with the LM method. The incidence of carotid puncture in the LM group in this study was $11.9 \%$ which is within the $8.3-13 \%$ range reported in previous studies (Karakitsos et al., 2006; Serafimidis et al., 2009). The incidence of carotid puncture in the US group is significantly lower than the LM group, confirming the results of previous studies (Denys et al., 1993; Karakitsos et al., 2006). Sazdov et al. (2017) reported a $14.5 \%$ complication rate in the landmark guided techniques versus a $4 \%$ rate when using ultrasound (Sazdov et al., 2017). Other studies have reported similar significantly lower complication rates in the ultrasound guided method (Froehlich et al., 2009; Lamperti et al., 2012; Turker et al., 2009). The reduction in the rates of complications is due to the clear anatomical visualization of the potential CVC site. In addition, thrombosis in veins can be visualised and those veins can be avoided (Hind et al., 2003). Moreover, our resultsare comparable to other studies who have reported that the rates of hematomas using anatomical landmarks were (3.3-8.4\%) of patients (Kara-Kitsos et al., 2006; Miller et al., 2002; Serafimidis et al., 2009). The incidence of carotid puncture in the LM group in this study was $11.9 \%$ which is within the $8.3-13 \%$ range reported in previous literature (Karakitsos et al., 2006; Serafimidis et al., 2009). The incidence of carotid puncture in the US group is significantly lower than the LM group, confirming the results of previous literature (Denys et al., 1993; Karakitsos et al., 2006). The 
reduction in the rates of complications is due to the clear anatomical visualization of the potential CVC site. In addition, thrombosis in veins can be visualised and those veins can be avoided (Hind et al., 2003). Moreover, ourresult in the study is in comparable to other studies who reported that the rates of hematomas using anatomical landmarks have been reported in 3.3-8.4\% of patients (Karakitsos et al., 2006; Miller et al., 2002; Serafimidis et al., 2009).

The current study did not find significant difference between inexperienced physicians and experienced physicians in the number of attempts and time needed for insertion of a catheter in both techniques, indicating that inexperienced physicians are capable of performing the procedure after having the required training and supervision for CVC using US-guided technique. Similar results have also been reported by Miller et al. (2002) study.

The results of our study as it has been mentioned earlier are in line with previous studies conducted on non-Jordanian populations. The US-guided technique can be considered as a key factor in achieving better outcomes in Jordanian patients requiring CVC. The widespread application of ultrasound guided CVC would be beneficial in reducing insertion time, number of attempts and complications rates. This will ultimately will improve patient's outcomes, improve the quality of delivered medical care and reduce the overall cost of delivering medical care (Turker et al., 2009).

The main limitation of this study is that the sample was not randomised as it was a retrospective investigation for patients undergoing $\mathrm{CVC}$ at the OR who were enrolled in the US group and ICU patients who were enrolled in the LM group. Future multicenter prospective studies with comparable randomized samples are recommended in order to investigate different approaches on a larger scale.

\section{CONCLUSION}

Ultrasound guided CVC leads to lower complication rates, shorter duration of insertion and shorter duration of the whole procedure. Introducing US-guided catheterization would allow physicians to safely complete the procedure in less time and will decrease the likelihood of developing medical complications, justifying the cost needed for implementing the ultra sound technique. In addition both experienced and inexperienced physicians are able to successfully perform CVC under ultra sound guidance.

\section{REFERENCES}

Bannon, M.P., S.F. Heller and M. Rivera, 2011. Anatomic considerations for central venous cannulation. Risk Manage. Healthcare Policy, 4: 27-39.
Denys, B.G., B.F. Uretsky and P.S. Reddy, 1993. Ultrasound-assisted cannulation of the internal jugular vein: A prospective comparison to the external landmark-guided technique. Circulation, 87: 1557-1562.

Digby, S., 1994. Fatal respiratory obstruction following insertion of a central venous line. Anaesthesia, 49: 1013-1014.

Emerman, C.L., E.M. Bellon, T.W. Lukens, T.E. May and D. Effron, 1990. A prospective study of femoral versus subclavian vein catheterization during cardiac arrest. Annal. Emergency Med., 19: 26-30.

Fragou, M., A. Gravvanis, V. Dimitriou, A. Papalois and G. Kouraklis et al., 2011. Real-time ultrasound-guided subclavian vein cannulation versus the landmark method in critical care patients: A prospective randomized study. Crit. Care Med., 39: 1607-1612.

Froehlich, C.D., M.R. Rigby, E.S. Rosenberg, R. Li, P.L.J. Roerig, K.A. Easley and J.A. Stockwell, 2009. Ultrasound-guided central venous catheter placement decreases complications and decreases placement attempts compared with the landmark technique in patients in a pediatric intensive care unit. Crit. Care Med., 37: 1090-1096.

Ge, X., R. Cavallazzi, C. Li, S.M. Pan, Y.W. Wang and F.L. Wang, 2012. Central venous access sites for the prevention of venous thrombosis, stenosis and infection. Cochrane Database Syst. Rev., Vol. 3, 10.1002/14651858.CD004084.pub3

Hind, D., N. Calvert, R. McWilliams, A. Davidson, S. Paisley, C. Beverley and S. Thomas, 2003. Ultrasonic locating devices for central venous cannulation: Meta-analysis. Bmj., 327: 361-364.

Hosokawa, K., N. Shime, Y. Kato and S. Hashimoto, 2007. A randomized trial of ultrasound image-based skin surface marking versus real-time ultrasound-guided internal jugular vein catheterization in infants. Anesthesiology, 107: 720-724.

Karakitsos, D., N. Labropoulos, E. De Groot, A.P. Patrianakos and G. Kouraklis et al., 2006. Real-time ultrasound-guided catheterisation of the internal jugular vein: A prospective comparison with the landmark technique in critical care patients. Crit Care, Vol. 10, 10.1186/cc5101

Koski, E.M., M. Suhonen and M.A. Mattila, 1992. Ultrasound-facilitated central venous cannulation. Crit. Care Med., 20: 424-426.

Lamperti, M., A.R. Bodenham, M. Pittiruti, M. Blaivas and J.G. Augoustides et al., 2012. International evidence-based recommendations on ultrasound-guided vascular access. Intensive Care Med., 38: 1105-1117. 
McGee, D.C. and M.K. Gould, 2003. Preventing complications of central venous catheterization. N. Engl. J. Med., 348: 1123-1133.

Miller, A.H., B.A. Roth, T.J. Mills, J.R. Woody, C.E. Longmoor and B. Foster, 2002. Ultrasound guidance versus the landmark technique for the placement of central venous catheters in the emergency department. Acad. Emergency Med., 9: 800-805.

Palepu, G.B., J. Deven, M. Subrahmanyam and S. Mohan, 2009. Impact of ultrasonography on central venous catheter insertion in intensive care. Indian J. Radiol. Imaging, 19: 191-198.

Prabhu, M.V., D. Juneja, P.B. Gopal, M. Sathyanarayanan, S. Subhramanyam and S. Gandhe, 2010. Ultrasound-guided femoral dialysis access placement: A single-center randomized trial. Clin. J. Am. Soc. Nephrol., 5: 235-239.

Randolph, A.G., D.J. Cook, C.A. Gonzales and C.G. Pribble, 1996. Ultrasound guidance for placement of central venous catheters: A meta-analysis of the literature. Crit. Care Med., 24: 2053-2058.

Richards, M.J., J.R. Edwards, D.H. Culver and R.P. Gaynes, 1999. Nosocomial infections in medical intensive care units in the United States. National Nosocomial Infections Surveillance System. Crit. Care Med., 27: 887-892.

Sazdov, D., M.J. Srceva and Z.N. Todorova, 2017. Comparative analysis of ultrasound guided central venous catheterization compared to blind catheterization. Prilozi, 38: 107-114.
Scott, W.L., 1988. Complications associated with central venous catheters: A survey. Chest, 94: 1221-1224.

Serafimidis, K., G.H. Sakorafas, G. Konstantoudakis, K. Petropoulou and G.P. Giannopoulos et al., 2009. Ultrasound-guided catheterization of the internal jugular vein in oncologic patients; comparison with the classical anatomic landmark technique: A prospective study. Int. J. Surg., 7: 526-528.

Sznajder, J.I., F.R. Zveibil, H. Bitterman, P. Weiner and S. Bursztein, 1986. Central vein catheterization: Failure and complication rates by three percutaneous approaches. Arch. Internal Med., 146: 259-261.

Troianos, C.A., D.R. Jobes and N. Ellison, 1991. Ultrasound-guided cannulation of the internal jugular vein a prospective, randomized study. Anesth. Analg., 72: 823-826.

Turker, G., F.N. Kaya, A. Gurbet, H. Aksu, C. Erdogan and A. Atlas, 2009. Internal jugular vein cannulation: An ultrasound-guided technique versus a landmark-guided technique. Clinics, 64: 989-992.

Wu, S.Y., Q. Ling, L.H. Cao, J. Wang, M.X. Xu and W.A. Zeng, 2013. Real-time two-dimensional ultrasound guidance for central venous cannulation a meta-analysis. J. Am. Soc. Anesthesiologists, 118: 361-375.

Yonei, A., T. Nonoue and A. Sari, 1986. Real-time ultrasonic guidance for percutaneous puncture of the internal jugular vein. Anesthesiology, 64: $830-831$. 